\title{
Comparison of Elemental Performance for Stress Concentration Factor
}

\author{
Mrunal Kudale ${ }^{*}$, NandanSathe ${ }^{\#}$, Shubham Kale" , S.P.Wadkar" and S.N.Hawaldar" \\ \#Mechanical Department, M.I.T.C.O.E.,Kothrud,Pune, India
}

Accepted 03 March 2016, Available online 15 March 2016, Special Issue-4 (March 2016)

\begin{abstract}
Rectangular filleted bar or bracket is used to support horizontal shaft from a pillar where there is no wall in the way of wheels or pulleys on the shaft.It is also used in aerospace and automobile industries for supporting the structures like batteries and sensors in automobile for fixing the body to chassis and for fixing the auxiliaries. After the roofing work is finished, the overhang of sheets is supported by brackets. The railings around walkways and louvers provided for ventilation in a shed system are also supported by brackets. Also Square plate with hole is a frequently used component. Thus their finite element analysis plays important role. In this paper for Rectangular filleted bar, the effect of different types of elements used while meshing on FEA result is studied. Also for Square Plate effect of number of nodes on FEA results is studied.
\end{abstract}

Keywords: stress concentration factor(SCF), Plate with hole, Rectangular filleted bar

\section{Introduction}

The finite element methods (FEM), sometimes referred to as finite element analysis (FEA), are based on an idea of discretizing a physical domain into small manageable pieces and applying governing equations of the domain to these places which will lead to linear algebraic system of equations.

Types of elements:

1. 1D elements

2. 2D elements(triangular, rectangular)

3. 3D elements(tetrahedral, hexahedral)

Here we have used 2D elements for meshing.

Displacement functions for three-noded triangular element:

$u(x, y)=a_{1}+a_{2} x+a_{3} y$

$v(x, y)=a_{4}+a_{5} x+a_{6} y$

Shape functions for three-noded triangular element:

$$
\mathrm{N}_{\mathrm{i}}=\frac{1}{2 A}\left(\alpha_{\mathrm{i}}+\beta_{\mathrm{i}} \mathrm{x}+\gamma_{\mathrm{i}} \mathrm{y}\right)
$$

$\mathrm{N}_{\mathrm{j}}=\frac{1}{2 A}\left(\alpha_{\mathrm{j}}+\beta_{\mathrm{j}} \mathrm{x}+\gamma_{\mathrm{j}} \mathrm{y}\right)$

$$
\mathrm{N}_{\mathrm{m}}=\frac{1}{2 A}\left(\alpha_{\mathrm{m}}+\beta_{\mathrm{m}} \mathrm{x}+\gamma_{\mathrm{m}} \mathrm{y}\right)
$$

Where

$\alpha_{\mathrm{i}}=\mathrm{x}_{\mathrm{j}} \mathrm{y}_{\mathrm{m}}-\mathrm{y}_{\mathrm{j}} \mathrm{x}_{\mathrm{m}}$

$\beta_{\mathrm{i}}=\mathrm{y}_{\mathrm{j}}-\mathrm{y}_{\mathrm{m}}$

$\gamma_{\mathrm{i}}=\mathrm{x}_{\mathrm{m}}-\mathrm{x}_{\mathrm{j}}$

$\alpha_{\mathrm{i}}=\mathrm{x}_{\mathrm{j}} \mathrm{y}_{\mathrm{m}}-\mathrm{y}_{\mathrm{j}} \mathrm{x}_{\mathrm{m}}$

$\beta_{\mathrm{i}}=\mathrm{y}_{\mathrm{j}}-\mathrm{y}_{\mathrm{m}}$

$$
\begin{aligned}
& \gamma_{\mathrm{i}}=\mathrm{x}_{\mathrm{m}}-\mathrm{x}_{\mathrm{j}} \\
& \alpha_{\mathrm{i}}=\mathrm{x}_{\mathrm{j}} \mathrm{y}_{\mathrm{m}}-\mathrm{y}_{\mathrm{j}} \mathrm{x}_{\mathrm{m}} \\
& \beta_{\mathrm{i}}=\mathrm{y}_{\mathrm{j}}-\mathrm{y}_{\mathrm{m}} \\
& \gamma_{\mathrm{i}}=\mathrm{x}_{\mathrm{m}}-\mathrm{x}_{\mathrm{j}} \\
& \text { Displacement funct } \\
& \text { element: } \\
& \mathrm{u}(\mathrm{x}, \mathrm{y})=\mathrm{a}_{1}+\mathrm{a}_{2} \mathrm{x}+\mathrm{a}_{3} \\
& \mathrm{v}(\mathrm{x}, \mathrm{y})=\mathrm{a}_{5}+\mathrm{a}_{6} \mathrm{X}+\mathrm{a}_{7} \\
& \text { Shape function for } \mathrm{f} \\
& \mathrm{N}_{1}=\frac{(b-x)(h-y)}{4 b h} \\
& \mathrm{~N}_{2}=\frac{(b+x)(h-y)}{4 b h} \\
& \mathrm{~N}_{3}=\frac{(b+x)(h+y)}{4 b h} \\
& \mathrm{~N}_{4}=\frac{(b-x)(h+y)}{4 b h}
\end{aligned}
$$$$
\text { Displacement functions for four-noded triangular }
$$$$
u(x, y)=a_{1}+a_{2} x+a_{3} y+a_{4} x y
$$$$
v(x, y)=a_{5}+a_{6} x+a_{7} y+a_{8} x y
$$

Shape function for four-noded rectangle element:

\section{Problem statement}

The problems we have taken for analysis are 2D rectangular filleted barand plate with hole made of steel (Elasticity modulus $(\mathrm{E})=210 \mathrm{GPa}$, Modulus of rigidity $(\mathrm{G})=81 \mathrm{GPa}$, Density $\left.(\rho)=7.9 \mathrm{e}-9 \mathrm{~kg} / \mathrm{mm}^{3}\right)$. Poission's ratio $(v): 0.3$

\section{For rectangular filleted bar}

Load applied: $1000 \mathrm{~N}$

Cross sectional area $(A)=200 \mathrm{~mm}^{2}$ 
Thickness: $5 \mathrm{~mm}$

\section{For plate with hole}

Load applied: $10000 \mathrm{~N}$

Cross sectional area $(A)=9500 \mathrm{~mm}^{2}$

Thickness: $10 \mathrm{~mm}$

\section{Solution}

\section{By Analytical Method}

For rectangular filleted bar

Max. stress at fixed end:

$\sigma=5 \mathrm{MPa}$

Max. displacement:

$\delta=0.2380 \mathrm{e}-02 \mathrm{~mm}$

$\mathrm{k}_{\mathrm{t}}=1.6$

Stress at fillet:

$\mathrm{k}_{\mathrm{t}} * \sigma=8 \mathrm{MPa}$

For plate with hole:

Max. stress at fixed end:

$\sigma=1.0526 \mathrm{MPa}$

$\mathrm{k}_{\mathrm{t}}=3$

Max. stress near hole:

$\mathrm{k}_{\mathrm{t}} * \sigma=3 * 1.0526 \mathrm{MPa}$

$=3.1578 \mathrm{MPa}$

Max. displacement:

$$
\delta=5.01253 \mathrm{e}-03 \mathrm{~mm}
$$

\section{Finite element modeling}

\section{For rectangular filleted bar}

1) Mixed Mesh

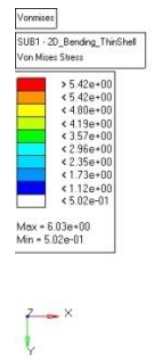

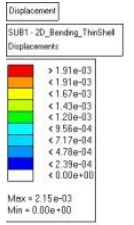
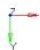

Fig.2 Displacement

Number of quadrilateral elements $=304$ Number of trianular elements $=4$

2) Triangular Mesh

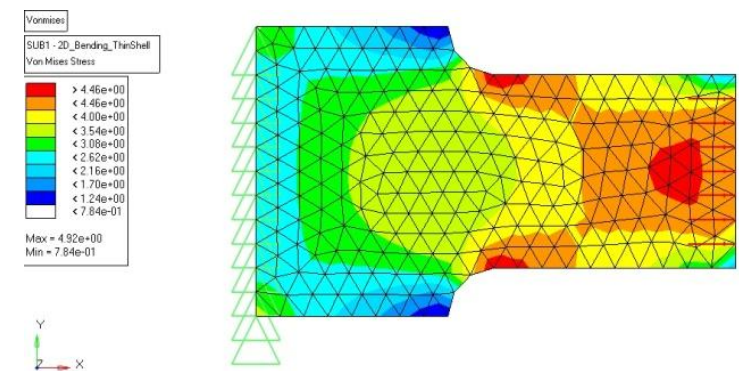

Fig.3 Vonmises Stress
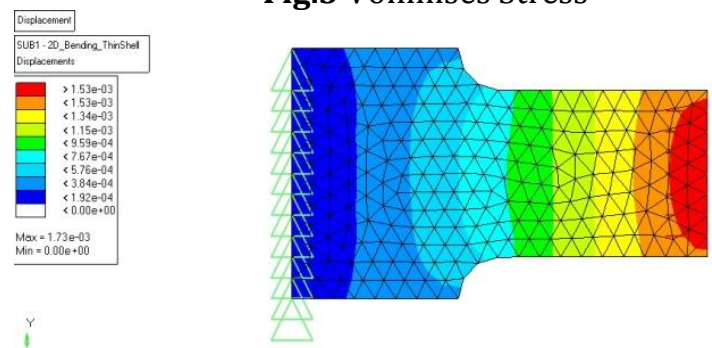

Fig.4 Displacement

Number of triangular elements $=432$

3) Mapped Mesh

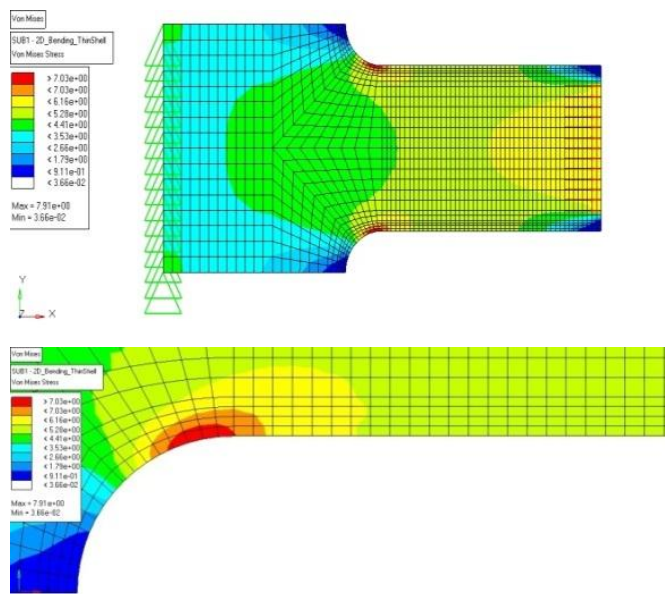

Fig.5 Vonmises Stress

Fig.1 Vonmises Stress 

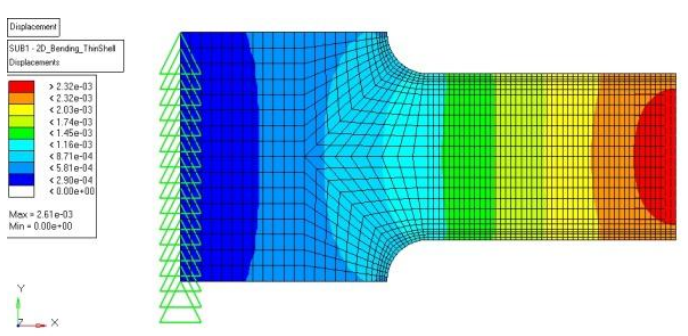

Fig.6 Displacemen

Number of quadrilateral elements $=1728$

\section{For plate with hole}

1) For 32 nodes
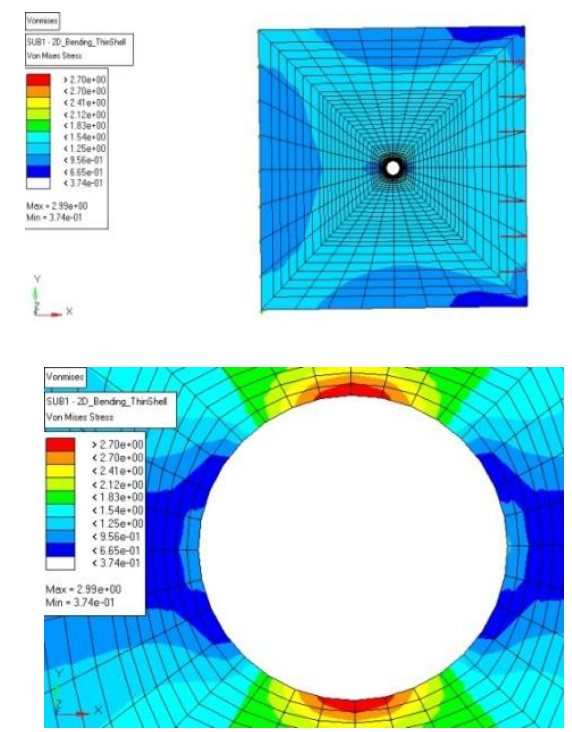

Fig.7 Vonmises Stress

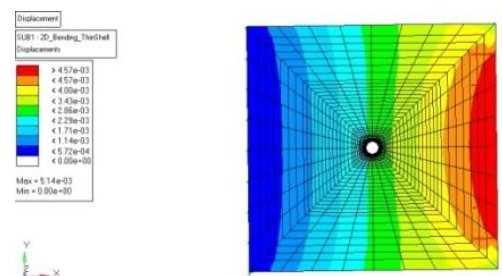

Fig.8 Displacement

Number of elements $=1152$

2) For 64 nodes

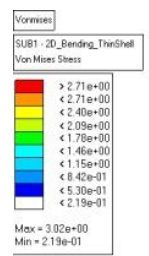

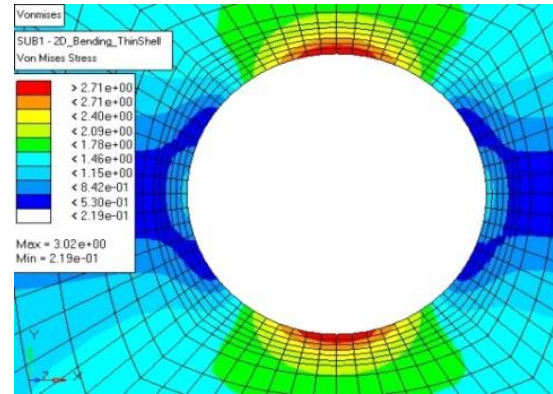

Fig.9 Vonmises Stress
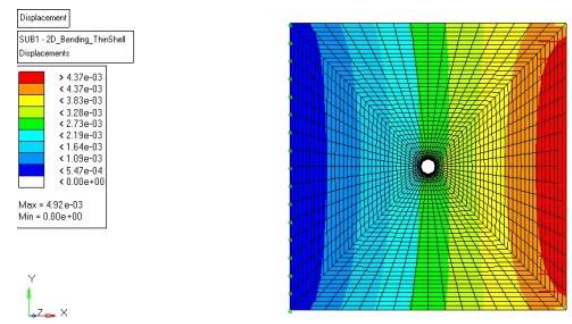

Fig.10 Displacement

Number of elements $=2624$

3) For 128 nodes
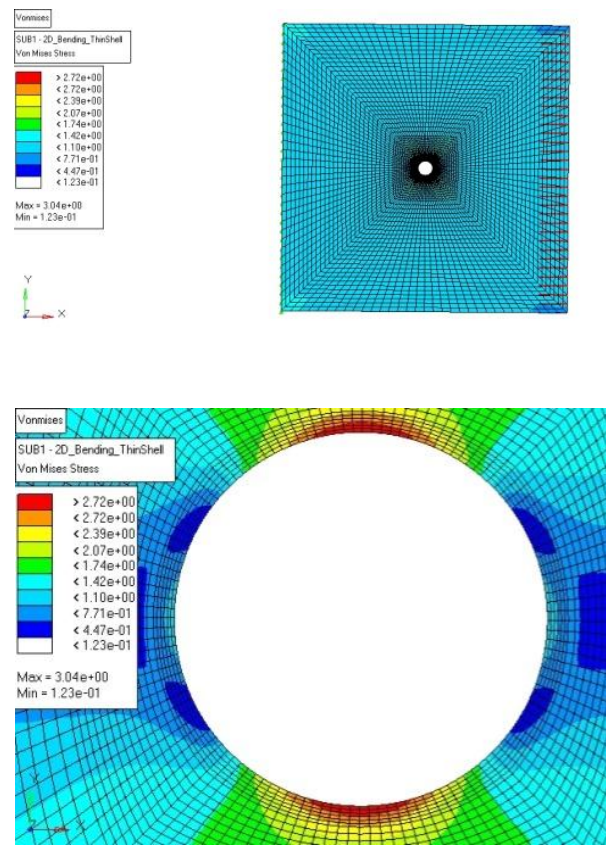

Fig.11 Vonmises Stress
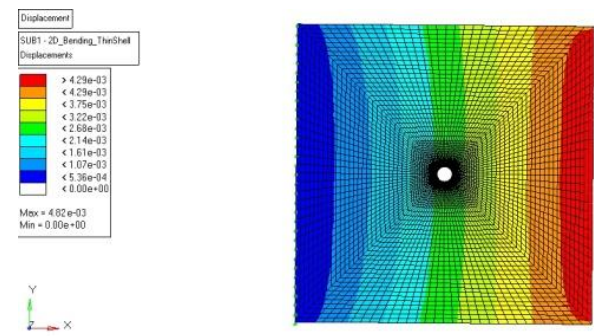

Fig.12 Displacement 
Number of elements $=7552$

\section{FEA Results}

For Rectangular filleted bar

\begin{tabular}{|c|c|c|c|c|}
\hline Mesh Type & Exact & Mixed & Triangular & Mapped \\
\hline Max. displacement (mm) & $0.2380 \mathrm{e}-02$ & $0.215126 \mathrm{e}-02$ & $0.215846 \mathrm{e}-02$ & $0.261 \mathrm{e}-02$ \\
\hline Max. stress at Fixed End (MPa) & 8 & 6.34500 & 6.93875 & 7.91 \\
\hline
\end{tabular}

For Plate with Hole

\begin{tabular}{|c|c|c|c|c|}
\hline No. of nodes & Exact & $\mathbf{3 2}$ & $\mathbf{6 4}$ & $\mathbf{1 2 8}$ \\
\hline Max. displacement (mm) & $5.012 \mathrm{e}-03$ & $5.14 \mathrm{e}-03$ & $4.92 \mathrm{e}-03$ & $4.82 \mathrm{e}-03$ \\
\hline Max. stress at Fixed End (MPa) & 3.1578 & 2.99 & 3.02 & 3.04 \\
\hline
\end{tabular}

\section{Conclusion}

It can be observed from table that for Rectangular filleted bar,Ruled mesh has given closest results to analytical results and mixed mesh has most deviation. For Square plate with hole, we obtain closer results to Analytical with increase in number of nodes. Thus Elemental Performance is better for quadrilateral nodes and thus are preferred for analysis.

\section{References}

Daryl L. Logan-A First Course in the Finite Element MethodCengag00e Learning (2011)

Richard Budynas, Keith Nisbett - Shigley's Mechanical Engineering Design, 10 th Edition - McGraw- Hill 